\title{
Switch From a ZDV/3TC-BAsed Regimen to a Completely Once Daily (QD) Regimen of Emtricitabine/Tenofovir DF Fixed Dose Combination plus a Third QD Agent (SONET'T)
}

\author{
K. Arasteh ${ }^{1}$, L. Weitner ${ }^{2}$, S. Fenske 2 , B. Kuhlmann ${ }^{3}$, M. Freiwald ${ }^{4}$, R. Ebrahimi ${ }^{5}$, L. Gallo ${ }^{5}$, \\ B. Ranneberg ${ }^{5}$, T. Mertenskoetter ${ }^{5}$ \\ ${ }^{1}$ Epimed, Berlin, ${ }^{2}$ IPM Study Center, Hamburg, ${ }^{3}$ Private Practice, Hannover, ${ }^{4}$ Private Practice, Berlin, ${ }^{5}$ Gilead Sciences, Martinsried, \\ Germany
}

\begin{abstract}
Objectives: To assess the efficacy and safety of a treatment switch from a twice-daily (BID) regimen containing zidovudine (ZDV) and lamivudine (3TC) plus a third agent to a once daily (QD) regimen containing the fixed-dose combination of tenofovir DF/emtricitabine (TDF/FTC, Truvada $\left.{ }^{\circledR}\right)$ plus a divergent third QD agent in HIV-1 infected patients.

Methods: Prospective, 48-week, non-randomised, single-group, open-label, study. Fifty-one patients on stable ZDV/3TC-containing HAART, with HIV-1 RNA $<50$ copies $/ \mathrm{ml}$ and $\mathrm{CD} 4+$ T-cell count $>50$ cells $/ \mu \mathrm{l}$, were switched to TDF/FTC plus a third agent. Plasma HIV-1 RNA, CD4+ and CD8+ T-cell counts were assessed at baseline and weeks 4, 12, 24, 36 and 48 postswitch.
\end{abstract}

Results: During the 48-week study, 10 patients discontinued prematurely, including three due to adverse events (AEs). At week 48, plasma HIV-1 RNA was $<50$ copies $/ \mathrm{ml}$ in 40 patients $(78.4 \%)$. No patient experienced virological failure (defined as HIV-1 RNA $\geq 50$ copies $/ \mathrm{ml}$ at two consecutive post-baseline measurements) during the study. Immunologic control was maintained, with no significant changes in CD4+ or CD8+ T-cell counts. A statistically significant improvement from baseline in haemoglobin level was observed at week 48 (median change $0.8 \mathrm{~g} / \mathrm{dl}$; $p<0.001)$. There was also a statistically significant decrease in total cholesterol concentration at week 48 $(-26.0 \mathrm{mg} / \mathrm{dl} ; \mathrm{p}=0.001)$ in a subset of patients $(\mathrm{n}=$ 22) entering the study with elevated total cholesterol. Treatment was well tolerated and no treatment-related grade 3 or 4 AEs were seen.

Conclusions: Results from this study support switching from a ZDV/3TC-containing HAART regimen to a completely QD regimen of TDF/FTC plus a third agent. Virologic and immunologic control are maintained, with apparent benefits in haemoglobin.

Key words: HAART; tenofovir DF; emtricitabine; combination therapy; once-daily

\section{INTRODUCTION}

The goal of antiretroviral therapy (ART) in type 1 HIV (HIV-1) is to maintain health and well-being with minimum drug side effects [1]. This is usually achieved by suppressing viral replication below detectable levels for as long as possible. In addition, keeping the viral titre at low levels helps to prevent the development of resistant viral strains [1]. Advances in therapy, such as the introduction of highly active ART (HAART), have greatly improved the prognoses of patients infected with HIV [2-4]. HAART typically includes a combination of three antiretroviral agents, including two nucleos(t)ide reverse transcriptase inhibitors (NRTIs/NtRTIs) with a non-nucleoside reverse transcriptase inhibitor (NNRTI) or a protease inhibitor (PI), which together are able to suppress HIV-1 to below detectable levels [1].

Despite the introduction of HAART, treatment is currently unable to completely eradicate HIV-1 [5, 6]. Therefore, successful, long-term efficacy is greatly dependent on patient adherence to the prescribed HAART regimen. Good adherence increases the likelihood of sustained virological control and reduces HIV-1-related morbidity and mortality, while poor adherence can lead to periods of viral replication and possible development of resistance to one or more of the drugs [7]. Therefore, the use of simplified, preferably once daily regimens with a low pill burden may be able to improve adherence $[8,9,10,11]$.

At initiation of the present study, first-line HIV-1 treatment for most patients in Germany was fixeddose ZDV/3TC complemented by an NNRTI or a boosted PI. Trials using this regimen have demonstrated successful viral suppression in addition to sustained increases in CD4+ T-cell counts, which restored some level of immune function to patients with HIV-1 infection [12]. However, the ZDV/3TC combination has limitations: it must be dosed twice daily (BID) due to the short half-life of ZDV and, while 3TC shows good tolerability, ZDV is known to cause a number of adverse effects, such as anaemia, fatigue and nausea [12]. Medication side effects including metabolic and morphological changes with HAART are one of the factors associated with poor adherence and shorter durability of first-line HAART [10, 13, 14].

There is a clinical need for potent regimens that simplify adherence through once daily (QD) dosing, offer good tolerability and fit into the daily life of patients living with HIV-1 infection. The fixed-dose combination of emtricitabine/tenofovir DF (Truva- 
$\mathrm{da}\left({ }^{\circledR}\right)$, as a dual-NRTI backbone, offers the possibility of a completely QD regimen. At the time this trial was started, the fixed dose combination of TDF/FTC was not yet licensed in the European Union, and data on the possibility of switching patients on stable HAART to TDF/FTC did not exist. This Phase III proof of concept study was designed to address the question of whether patients receiving a stable ZDV/3TC containing regimen can be switched to a completely QD TDF/FTC-containing regimen.

\section{PATIENTS AND Methods}

This prospective, single-group, open-label, 48-week, study enrolled 52 patients (one patient was excluded due to a high baseline HIV-1 RNA concentration). All patients had HIV-1 infection and were on a stable ZDV/3TC-containing BID regimen for at least the previous 3 months, with full viral suppression (HIV-1 RNA $<50$ copies $/ \mathrm{ml}$ ) and CD4+ T-cell counts $>50$ cells $/ \mu$ l. Viral load was determined locally to avoid screening failures, which may occur when switching the diagnostic system and to compare historical values with values during the study in patients with virologic failure. Patients were selected for the study if they were experiencing side effects ( $38 \%$ of patients), if it was deemed they would benefit from a QD regimen (50\%) or both $(12 \%)$. Patients were mainly male $(92 \%)$, with median age of 40.0 years (range 19-69 years), mean body mass index of $23.6 \mathrm{~kg} / \mathrm{m} 2$ and mean duration on a ZDV/3TC-containing regimen of 3.75 years.

The protocol was approved by the appropriate ethical committees and all patients signed an informed consent form. At baseline, patients switched from their current BID regimen to a QD regimen containing TDF/FTC and a third QD agent. The third agent was efavirenz for $30(58 \%)$ patients, nevirapine for 13 $(25 \%)$, a boosted protease inhibitor for $7(13 \%)$ and abacavir (recorded as a protocol violation) for $2(4 \%)$ patients. Divergent HAART was mandatory, i.e. the third drug had to be from the NNRTI or PI antiretroviral classes. Patients were assessed at baseline and weeks 4, 12, 24, 36 and 48. For patients withdrawn prematurely, a discontinuation visit took place as soon as possible.

HIV-1 RNA concentrations were measured using reverse transcriptase PCR (Roche Amplicor HIV-1
Monitor or COBAS AmpliPrep/COBAS TaqMan HIV-1 test). The primary endpoint was the proportion of patients who maintained the target HIV-1 RNA concentration of $<50$ copies/ml at week 48 , consistent with guidelines for viral suppression. Secondary endpoints included proportion of patients with HIV-1 RNA $<50$ copies/ml by study visit and changes in CD4+ and CD8+ T-cell counts. Virological failure was defined as HIV-1 RNA $\geq 50$ copies/ml for two consecutive post-baseline measurements. Safety included adverse event (AE) reporting and laboratory assessments. Serum creatinine concentration was used to estimate glomerular filtration rate and creatinine clearance by the Cockcroft-Gault method. Quality of Life was assessed by SF-12 Health Survey at baseline and weeks 12, 24 and 48 .

The primary analysis used an approach where any missing value was considered to be a treatment failure. Secondary analyses used approaches including missing $=$ excluded and early discontinuation $=$ failure. Statistical significance of changes from baseline was assessed using Wilcoxon signed rank tests.

\section{RESULTS}

The full 48 weeks of treatment was completed by $41 / 51(80.4 \%)$ patients. Early discontinuations were due to AE/intercurrent illness in three cases, protocol violation in three cases, patient decision in two cases, loss to follow-up in one case and non-compliance in one case.

At week 48, plasma HIV-1 RNA was $<50$ copies $/ \mathrm{ml}$ for $78 \%$ of patients $(40 / 51)$ using the missing $=$ failure approach. Using the missing = excluded approach, the 40 patients translated to $98 \%$ (40/41 patients). Numbers of patients with HIV-1 RNA $<50$ copies $/ \mathrm{ml}$ at each time point are shown in Table 1 by reason for switching to the TDF/FTC plus third agent QD regimen. There were no obvious differences between reasons for switching treatment. HIV-1 RNA $>50$ copies $/ \mathrm{ml}$ at any time was observed for two patients at week 24, who had switched therapy to simplify the regimen and had HIV-1 RNA concentrations of 50 and 59 copies/ml (both stayed on therapy and HIV-1 RNA was re-suppressed), and for one patient at week 48 (HIV-1 RNA $=254$ copies $/ \mathrm{ml}$ ) who had switched due to side effects. No patient experienced

Table 1. Numbers of patients with plasma HIV-1 RNA concentration $<50$ copies/ml at each study visit, by reason for switch to TDF/FTC (n (\%)).

Reason for switch of therapy

Side effects* $(\mathrm{N}=19) \quad$ Simplify regimen $(\mathrm{N}=26) \quad$ Both $(\mathrm{N}=6) \quad$ Total $(\mathrm{N}=51)$

\begin{tabular}{|c|c|c|c|c|}
\hline Week 4 & 18 (95) & $25(96)$ & $5(83)$ & $48(94)$ \\
\hline Week 12 & $19(100)$ & $23(88)$ & $5(83)$ & $47(92)$ \\
\hline Week 24 & $19(100)$ & $20(77)$ & $4(67)$ & $43(84)$ \\
\hline Week 36 & $18(95)$ & $18(69)$ & $3(50)$ & $39(76)$ \\
\hline Week 48 & $15(79)$ & $21(81)$ & $4(67)$ & $40(78)$ \\
\hline
\end{tabular}

* Side effects most commonly reported were: gastrointestinal disorders $(\mathrm{n}=12)$, lipodystrophy $(\mathrm{n}=7)$ and anaemia $(\mathrm{n}=3)$ 
Table 2. Change from baseline in T-cell counts and laboratory results at week 48; values are medians with interquartile ranges.

\begin{tabular}{lccc}
\hline & Baseline & Change to week 48 & $p$-value* \\
\hline CD4+ count $($ cells $/ \mu \mathrm{l})$ & $526(317: 774)$ & $30(-72: 125)$ & 0.23 \\
CD8+ count $($ cells $/ \mu \mathrm{l})$ & $907(664: 1209)$ & $-80(-263: 91)$ & 0.16 \\
Haemoglobin $(\mathrm{g} / \mathrm{dl})$ & $14.8(14.1: 15.3)$ & $0.8(0.3: 1.3)$ & $-5.0(-32:+17)$ \\
Fasting total cholesterol $(\mathrm{mg} / \mathrm{dl})$ & $194.0(167: 225)$ & $-26.0(-39:-4)$ & 0.203 \\
Fasting total cholesterol $(\mathrm{mg} / \mathrm{dl})$ & $240.5(222: 251)$ & & 0.001 \\
in patients with baseline values & & $52(44: 59)$ & 0.094 \\
$\geq 200 \mathrm{mg} / \mathrm{dl}(\mathrm{n}=22)$ & $50.5(44: 59)$ & $-1.3(-13.9: 8.1)$ & 0.20 \\
Fasting $\mathrm{HDL}(\mathrm{mg} / \mathrm{dl})$ & $114(97: 127)$ & & \\
Creatinine clearance $(\mathrm{ml} / \mathrm{min})$ & &
\end{tabular}

* p-value for change from baseline using Wilcoxon signed rank test

virological failure (defined as two consecutive measurements of HIV1-RNA > 50 copies/ml) during the study.

There were no statistically significant changes from baseline in CD4+ and CD8+ T-cell counts throughout the study (Table 2). At baseline, five patients had CD4+ T-cell counts $\leq 200 \mathrm{cell} / \mu \mathrm{l}$, of whom one was lost to follow-up immediately after and four had an increase in CD4+ T-cell count to $>200$ cells $/ \mu$ by week 48. At week 48, all patients with available data had CD4+ T-cell counts $>200$ cells $/ \mu$ l.

Laboratory tests indicated a statistically significant $(\mathrm{p}<0.001)$ increase in haemoglobin levels at week 48 (Table 2). There was no apparent effect on renal function, measured by creatinine clearance at week 48. While there was no difference in total cholesterol or HDL looking at the median values in all treated patients, there was a statistically significant $(p=0.001)$ decrease in fasting total cholesterol concentration at 48 weeks in patients with total cholesterol $\geq 200$ $\mathrm{mg} / \mathrm{dl}$ at baseline. Grade 3 toxicity elevations of alanine aminotransferase were reported for two patients and grade 4 increase in asparate aminotransferase for one patient, and grade 3 or 4 decreases in blood glucose were seen in two patients. None of the grade $3 / 4$ toxicities were considered related to treatment.

Treatment-emergent AEs were reported for 45/52 patients $(87 \%)$, with the most frequent being nasopharyngitis $(60 \%)$, diarrhoea $(13 \%)$ and gastroenteritis $(10 \%)$. Most were deemed unrelated to treatment and only 13 AEs in seven patients were considered treatment-related by the study investigators (Table 3). Serious AEs were reported for three patients but were classified as unrelated to treatment (inguinal hernia, hepatic failure and depression). AEs resulted in discontinuation for three patients, of which two were considered related to treatment (renal pain and drug hypersensitivity).

No statistically significant changes from baseline in SF-12 (Version 1) physical or mental composite scores were reported during the study, Scores for these HIV infected subjects were similar to the general population, i.e., with mean scores of approximately 50 .
Table 3. Treatment-emergent adverse events related to TDF/ FTC (none of these events were Grade 3 or 4).

\begin{tabular}{lcc}
\hline & $\mathrm{n}$ & $\%$ \\
\hline $\begin{array}{l}\text { Number of patients experiencing any adverse } \\
\text { event related to TDF/FTC }\end{array}$ & 7 & $13 \%$ \\
Cardiac disorder (palpitation) & 1 & $2 \%$ \\
Gastrointestinal disorders (flatulence, faeces & & \\
discoloured) & 2 & $4 \%$ \\
Drug Hypersensitivity & 1 & $2 \%$ \\
Increased Amylase & 1 & $2 \%$ \\
Increased Creatine phosphokinase & 1 & $2 \%$ \\
Lactic Acid increased & 1 & $2 \%$ \\
Increased Lipase & 1 & $2 \%$ \\
Arthralgia & 1 & $2 \%$ \\
Nervous System Disorders (Dizziness, & 1 & $2 \%$ \\
paraesthesia) & & \\
Renal pain & 1 & $2 \%$ \\
Rash & 1 & $2 \%$ \\
\hline
\end{tabular}

\section{Discussion}

Despite the availability of potent antiretroviral therapies, many patients with HIV-1 infection are still unable to achieve or maintain adequate suppression of viral load, often because of incomplete adherence to the treatment regimen $[7,9,10,11]$. Total pill burden, dosing frequency and safety concerns have been identified as specific obstacles to achieving adherence $[8,9$, $10,11]$.

Switching regimens is a standard means of maintaining suppression of viral load and supporting immune function over time, particularly when the switch is to a simpler regimen $[15,16]$. However, every switch bears a risk of failure or changes in tolerability. In the present study, patients switched from a BID regimen to an entirely QD regimen. There were no cases of confirmed virological failure, defined as two consecutive HIV-1 RNA measurements $\geq 50$ copies $/ \mathrm{ml}$, and therefore, no testing for virological resistance was per- 
formed. The QD regimen of TDF/FTC plus a third agent was well tolerated in this study population and most AEs were considered unrelated to the study regimen. In the IT'T analysis, viral load was maintained below 50 copies in $78 \%$ of patients at week 48 . Ten patients discontinued the trial prematurely, two due to adverse events considered related to the study drug, and eight for other reasons (protocol violation, consent withdrawal, loss to follow up, non-compliance). This number may have been influenced by the fact that the fixed dose combination of TDF/FTC was licensed and commercially introduced during the study period.

Laboratory tests revealed an improvement in haemoglobin levels following the switch to a QD regimen of TDF/FTC plus a third agent. This possibly reflected the change from a ZDV-containing therapy, which has been reported to cause anaemia [17]; however, no patients were anaemic at baseline in this study. Previous studies have demonstrated an association between increased haemoglobin levels, even within the normal range, and improved energy and quality of life in AIDS patients [18]. HAART has been shown to produce dyslipidaemia in HIV-1 infected patients [19]. The present pilot study demonstrated a decrease in fasting total cholesterol after 48 weeks of switching from a ZDV/3TC-containing BID regimen to a TDF/FTC containing QD regimen in patients with elevated values at baseline. Additional information on changes in total cholesterol, HDL, LDL and triglycerides is available from the COMET study in which patients were switched from ZDV/3TC+EFV to TDF/FTC+ EFV [20], The results from this study showed statistically significant declines in all four parameters between baseline and week 24 .

There are some important limitations associated with this trial. The first and most obvious is the lack of a control group. The present study was designed as a proof of concept study to address whether it would be safe to switch virologically stable patients from ZDV/3TC to TDF/FTC, which was at that time not yet licensed in Europe. As such, SONETT enrolled only a relatively small number of patients and did not include any comparator treatment. However, comparison with baseline values indicated that patients who switched from a ZDV/3TC-containing BID regimen to a QD regimen of TDF/FTC plus a third QD agent maintained virologic suppression and immunologic function over the 48 week treatment period. In the meantime, results from an open-label, randomized switch trial (SWEET) [21] have been published, which show similar findings to those reported in SONETT.

The benefits of switching from a BID to a QD regimen could not be fully evaluated, as these patients, who had been successfully treated with a ZDV/3TCcontaining regimen for a median of 3.75 years, were adherent to their regimen. This may, together with the small numbers of patients, also explain the lack of a difference in quality of life between baseline and week 48. Some information on the possible benefits of switching to a complete QD regimen can be derived from COMET, which enrolled 402 patients: treatment satisfaction and absence of side effects changed significantly $(\mathrm{p}<0.001)$ between baseline and week 24 [20].
In summary, the results from the present pilot study support switching from a BID ZDV/3TC-containing regimen to a completely QD regimen of TDF/FTC plus a third agent. The results show maintained virologic and immunologic control consistent with similar switching strategies examined in the COMET and SWEET studies [20, 21]. TDF/FTC QD plus third agent regimen also appears to provide benefits in improved haemoglobin and total cholesterol profiles.

Acknowledgements: The study was supported by Gilead Sciences GmbH, Germany. The authors would like to thank Peter Bates, PhD, of IMC Medical Communication, UK, for help in preparation of the manuscript.

\section{REFERENCES}

1. Thaker HK, Snow MH: HIV viral suppression in the era of antiretroviral therapy. Postgrad Med J. 2003; 79: 36-42.

2. Panos G, Samonis G, Alexiou VG, Kavarnou GA, Charatsis G, Falagas ME: Mortality and morbidity of HIV infected patients receiving HAART: a cohort study. Curr HIV Res. 2008; 6: 257-260.

3. Palella FJ Jr, Baker RK, Moorman AC, Chmiel JS, Wood $\mathrm{KC}$, Brooks JT, Holmberg SD: Mortality in the highly active antiretroviral therapy era: changing causes of death and disease in the HIV outpatient study. J Acquir Immune Defic Syndr. 2006; 43: 27-34.

4. Sterne JAC, Herna_n MA, Ledergerber B, Tilling K, Weber R, Sendi P, Rickenbach M, Robins JM, Egger M: Long term effectiveness of potent antiretroviral therapy in preventing AIDS and death: a prospective cohort study. Lancet. 2005; 366: 378-384.

5. Kulkosky J, Bray S: HAART-persistent HIV-1 latent reservoirs: their origin, mechanisms of stability and potential strategies for eradication. Curr HIV Res. 2006; 4: 199-208.

6. Delobel P, Sandres-Saune K, Cazabat M, L'Faqihi FE, Aquilina C, Obadia M, Pasquier C, Marchou B, Massip P, Izopet J: Persistence of distinct HIV-1 populations in blood monocytes and naive and memory CD4 $\mathrm{T}$ cells during prolonged suppressive HAART. AIDS. 2005; 19: 1739-1750.

7. Paterson DL, Swindells S, Mohr J, Brester M, Vergis EN, Squier C, Wagener MM, Singh N: Adherence to protease inhibitor therapy and outcomes in patients with HIV infection. Ann Intern Med. 2000; 133: 21-30.

8. Stone VE, Hogan JW, Schuman P, Rompalo AM; Howard AA, Korkontzelou C, Smith DK: Antiretroviral Regimen Complexity, Self-Reported Adherence, and HIV Patients' Understanding of Their Regimens: Survey of Women in the HER Study. JAIDS, Vol. 28(2), 2001, pp. 124-131.

9. Murri R, Marcotullio S, Lupoli P, von Schloesser F: Is Once-Daily Regimen a Key Strategy for Improving Adherence to Antiretroviral Regimens? JAIDS, Vol. 42 (2), 2006, pp. 259-260.

10. Willig JH, Abroms S, Westfall AO, Routman J, Adusumilli S, Varshney M, Allison J, Chatham A, Raper JL, Kaslow RA, Saag MS, Mugavero MJ: Increased regimen durability in the era of once-daily fixed-dose combination antiretroviral therapy. AIDS 2008, 22:1951-1960

11. Parienti JJ, Bangsberg DR, Verdon R, Gardner EM. Better Adherence with Once-Daily Antiretroviral Regimens: A Meta-Analysis. CID 2009, 48: 484-488

12. DeJesus E, Herrera G, Teofilo E, Gerstoft J, Buendia CB, Brand JD, Brothers CH, Hernandez J, Castillo SA, Bonny T, Lanier ER, Scott TR: Abacavir versus zidovudine combined with lamivudine and efavirenz, for the treatment of 
antiretroviral-naive HIV-infected adults. Clin Infect Dis. 2004; 39: 1038-1046.

13. Chesney MA: Factors affecting adherence to antiretroviral therapy. Clin Infect Dis. 2000; 30(Suppl 2): S171-S176.

14. Chesney MA, Ickovics J, Hecht FM, Sikipa G, Rabkin J: Adherence: a necessity for successful HIV combination therapy. AIDS. 1999; 13(Suppl A): S271-S278.

15. Conway B: The role of adherence to antiretroviral therapy in the management of HIV infection. J Acquir Immune Defic Syndr. 2007; 45(Suppl 1): S14-S18.

16. Barrios A, Negredo E, Domingo P, Estrada V, Labarga P, Asensi V, Morales D, Santos J, Clotet B, Soriano V: Simplification therapy with once-daily didanosine, tenofovir and efavirenz in HIV-1-infected adults with viral suppression receiving a more complex antiretroviral regimen: final results of the EFADITE trial. Antvir Ther. 2005; 10: 825-832.

17. Huffam SE, Srasuebkul P, Zhou J, Calmy A, Saphonn V, Kaldor JM, Ditangko R: Prior antiretroviral therapy experience protects against zidovudine-related anaemia. HIV Med. 2007; 8: 465-471.

18. Semba RD, Martin BK, Kempen JH, Thorne JE, Wu AW: The impact of anemia on energy and physical functioning in individuals with AIDS. Arch Intern Med. 2005; 165: 2229-2236.

19. Riddler SA, Li X, Chu H, Kingsley LA, Dobs A, Evans R, Palella F, Visscher B, Chmiel JS, Sharrett A: Longitudinal changes in serum lipids among HIV-infected men on highly active antiretroviral therapy. HIV Med. 2007; 8: 280-287.
20. DeJesus E, Ruane P, McDonald C, Garcia F, Sharma S for the COMET Team: Impact of Switching Virologically Suppressed, HIV-1-Infected Patients from Twice-Daily FixedDose Zidovudine/Lamivudine to Once-Daily Fixed-Dose Tenofovir Disoproxil Fumarate/Emtricitabine. HIV Clin Trials 2008;9(2):103-114.

21. Moyle G, Fisher M, Reilly G, the SWEET Study Group: A randomized comparison of continued zidovudine plus lamivudine BID (Combivir, CBV) versus switching to tenofovir DF plus emtricitabine (Truvada, TVD) each plus efavirenz (EFV) in stable HIV infected persons: results of a planned 24 week analysis. 4th IAS Conference on HIV Pathogenesis, Treatment, and Prevention Sydney, Australia, July 2007 [Abstract WEPEB028].

Received: October 24, 2008 / Accepted: March 26. 2009

Address for correspondence:

Keikawus Arasteh, MD, PhD

EPIMED GmbH

c/o Vivantes-Auguste-Viktoria-Klinikum

Rubensstrasse 127

12157 Berlin

Germany

Tel.: $\quad+49307903$ 2581;

Fax: +4922897983 34;

E-mail: keikawus.arasteh@vivantes.de 